\title{
A microfluidic erythrocyte sedimentation rate analyzer using rouleaux formation kinetics
}

\author{
Ziya Isiksacan ${ }^{1} \cdot$ Mohammad Asghari $^{1} \cdot$ Caglar Elbuken $^{1}$ (I)
}

Received: 16 October 2016 / Accepted: 13 February 2017 / Published online: 24 February 2017

(C) Springer-Verlag Berlin Heidelberg 2017

\begin{abstract}
Red blood cell aggregation is an intrinsic property of red blood cells that form reversible stacked structures, also called rouleaux, under low shear rates. Erythrocyte sedimentation rate (ESR), commonly performed in clinics, is an indirect inflammation screener and a prognostic test for diseases. We have recently developed a microfluidic system for rapid measurement of ESR from $40 \mu \mathrm{l}$ whole blood employing the aggregation dynamics. In this work, we propose the use of an aggregation inducer, dextran polyglucose, for the preparation of multiple blood samples with differing aggregation dynamics. Using these samples, we characterized the performance of the system with three aggregation indices and under varying experimental conditions. Additionally, using the same underlying principle, we improved the system for ESR measurement using both venipuncture and fingerprick whole blood samples depending on the user needs. The results demonstrate that the system performs equally well with both samples, which validates the compatibility of the system for both laboratory and point-of-care applications where venous and capillary blood are the primary samples, respectively. The detailed characterization presented in this study legitimates the feasibility of the system for ultrafast and facile
\end{abstract}

This article is part of the topical collection "2016 International Conference of Microfluidics, Nanofluidics and Lab-on-a-Chip, Dalian, China" guest edited by Chun Yang, Carolyn Ren and Xiangchun Xuan.

Caglar Elbuken

elbuken@unam.bilkent.edu.tr

UNAM - National Nanotechnology Research Center, Institute of Materials Science and Nanotechnology, Bilkent University, 06800 Ankara, Turkey measurement of ESR in clinics and diverse off-laboratory settings.

Keywords Red blood cell · Erythrocyte sedimentation rate (ESR) · Aggregation · Dextran · Point-of-care · Microfluidics

\section{Introduction}

Red blood cells form face-to-face linear or branched structures, called as rouleaux, under static conditions or low shear rates in the presence of appropriate macromolecules (Baskurt et al. 2011a; Chien 1975; Merrill 1969; Neu and Meiselman 2007). This process is reversible, and cells can be dispersed individually when a disaggregating shear rate is applied to the cells (Baskurt et al. 2011b; Lim et al. 2010; Mehri et al. 2014; Shin et al. 2009). Aggregation is a critical factor for the determination of blood viscosity (Chien et al. 1967) and hematocrit level (Deng et al. 1993; Xu et al. 2008); hence, it is physiologically relevant to in vivo microcirculation of blood (Baskurt et al. 2011a; Baskurt and Meiselman 2013; Tuma et al. 2011). Red blood cell aggregation associated disorders include hypertension (Lominadze et al. 1998) and thrombosis (Andrews and Low 1999). In addition to (1) the shear forces applied to the cells (Baskurt et al. 2011b; Lim et al. 2010; Shin et al. 2009) and (2) the cellular factors (electrostatic repulsion, membrane strain) (Chien and Jan 1973; Jan and Chien 1973; Nash and Meiselman 1983; Rampling et al. 2004), the aggregation process is affected by the presence and concentrations of macromolecules in the suspending medium (fibrinogen or dextran) (Armstrong et al. 2001; Barshtein et al. 1998; Neu et al. 2008). Two hypotheses are proposed for the explanation of the aggregation dynamics, 
both without clear elucidation (Baskurt et al. 2011a): bridging and depletion (Baskurt et al. 2011a; Bishop et al. 2001; Neu et al. 2008; Rampling et al. 2004). The former claims that the cells clump due to the attractive forces caused by the adsorption of the macromolecules on the cell surfaces (Chien 1975). The latter proposes that aggregation occurs when macromolecule concentration near the cell surface is low (Asakura and Oosawa 1954). Aggregation of red blood cells can be quantified by photometric devices that monitor the interaction of cells with incident light passing through the sample (Baskurt et al. 1998, 2009, 2010; Hysi et al. 2012).

Indirect screening of inflammatory conditions such as rheumatism and tuberculosis is carried out in clinics by a simple routine test named erythrocyte sedimentation rate (ESR) (Hardwicke and Squire 1952; Tilted and Short 1999; Westergren 1921). This test is also a non-specific measure of some non-inflammatory disorders including heart failure and cancer (Andresdottir et al. 2003; Gillum et al. 1995; Johansson et al. 1992; Singh et al. 2014; Sox and Liang 1986). ESR expresses the separation rate of red blood cells from plasma in a vertically positioned tube (Baskurt et al. 2011a; Dima et al. 2016). The plasma height, which is read after $1 \mathrm{~h}$ of sample introduction to the tube, gives the ESR value. ESR values above $30 \mathrm{~mm} / \mathrm{h}$ are accepted to be in the clinical range and require further clinical investigation (Baskurt et al. 2011a). In the clinical settings, the common techniques performed to determine ESR are Wintrobe (Wintrobe and Landsberg 1935) and Westergren (Westergren 1921) methods differing based on the tube size and anticoagulant type (Bull and Brailsford 1972; Gilmour and Sykes 1951). The test is usually performed by a personnel in the clinics, but there are also automated benchtop products on the market (Plebani et al. 1998).

Microfluidic platforms hold great potential for health monitoring and diagnostics especially when there is need for rapid analysis using low sample volumes (Jiang et al. 2011; Li et al. 2012; Manage et al. 2011; Yang et al. 2014). In our previous work, we reported a microfluidic system for the measurement of ESR from aggregation process in 2 min using $40 \mu \mathrm{l}$ of whole blood (Isiksacan et al. 2016a). The system quantifies the rate of rouleaux formation and reports ESR, based on the relationship between ESR and red blood cell aggregation. Stokes' Law states that the sedimentation velocity of particles is proportional to their sizes (Stokes 1851). Since red blood cell aggregates are larger in size compared to individual cells, their sedimentation velocities are expected to be higher. This relationship was investigated for the realization of rapid ESR measurement from aggregation dynamics.

Herein, we further examined the underlying mechanism and the performance of this microfluidic system in an effort to address the practical aspects of this method, which is crucial when developing a truly sample-in result-out system. We used an aggregation inducer macromolecule (70 kDa Dextran) of different concentrations to change the aggregation dynamics of whole blood acquired from a single donor. We found a concentration interval where aggregation, thus ESR, increases linearly with dextran concentration. We have also demonstrated that bridging hypothesis can be used to explain the aggregation process. After finding the appropriate concentration levels, the performance of the microfluidic system was successfully demonstrated. Disaggregation of the cells within the microfluidic chips at the start of the test is critical, which is determined by the applied shear rate on the cells (Baskurt et al. 2011b; Lim et al. 2010; Mehri et al. 2014; Shin et al. 2009). We have shown computationally and experimentally that the choice/design of the mechanical unit, which is a pinch valve in this presented work, is critical to obtain adequate shear rate and therefore to perform a successful measurement. It was also shown that device orientation and vibrations do not alter the results of the ESR measurements. In addition, the microfluidic system was further improved to allow ESR measurement of venipuncture blood (suitable for clinical use) and fingerprick blood (suitable for on-site use) on the same chip. The performance of the improved system shows that this microfluidic platform is suitable for point-of-care ESR measurement, which can be performed by non-technical personnel in resource poor settings.

\section{Experimental}

\subsection{Materials}

$10 \mathrm{ml}$ whole blood was obtained from a healthy male donor at Bilkent University Health Center (Ankara, Turkey) into a vacuum tube containing ethylenediaminetetraacetic acid (EDTA; $1.5 \mathrm{mg} / \mathrm{ml}$ ) to prevent coagulation. The blood was centrifuged three times at $4000 \mathrm{rpm}$ for $4 \mathrm{~min}$, and the plasma portion was replaced with $1 \times$ PBS solution after each step. (1) After centrifugation, five micro centrifuge tubes $(\mathrm{T} 1, \ldots \mathrm{T} 5)$ were filled with $500 \mu \mathrm{l}$ of the PBS-suspended red blood cells. Then, $70 \mathrm{kDa}$ Dextran (Sigma-Aldrich) was added to the tubes at amounts of $0,2.5,5,7.5$, and $10 \mathrm{mg}$. Then, dextran was dissolved in the tubes by mixing the samples for 2 min with a pipette. (2) For red blood cell monitoring tests, after the centrifugation, a micro centrifuge tube was filled with $5 \mu$ of the PBS-suspended red blood cells and $125 \mu \mathrm{l}$ PBS. Then, $1 \mathrm{mg} 70 \mathrm{kDa}$ Dextran was added and dissolved by 2 min gentle mixing. 


\subsection{Theory}

Stokes' Law relates the settling velocity of a spherical particle in a medium to its size (Stokes 1851). There are three forces acting on a particle suspended in a medium: gravitational, buoyancy, and drag forces. Using these three forces, the particle velocity is found as:

$\vartheta=\frac{2 g}{9} \frac{\left(\rho_{\text {particle }}-\rho_{\text {medium }}\right)}{\mu} r^{2}$

where $\vartheta$ is particle settling velocity, $\rho$ is density, $g$ is gravitational acceleration constant, $\mu$ is medium dynamic viscosity, and $r$ is particle radius. This equation states that the settling velocity of a particle scales quadratically to its size.

\subsection{Microfluidic chip fabrication}

The material of choice for the microfluidic chips used in the ESR measurements was polymethyl methacrylate (PMMA) since it is transparent, bio-compatible, and mass-producible (Hong et al. 2010). It is also easier to obtain channels on a PMMA chip with high aspect ratios, compared to polydimethylsiloxane (PDMS). The chip used for ESR measurements using dextran-mediated blood consists of a $1 \mathrm{~mm}$ deep, $1 \mathrm{~mm}$ wide, and $50 \mathrm{~mm}$ long channel, with an inlet and outlet (single-channel chip). The chip used for whole blood tests consists of two parallel channels with $1 \mathrm{~mm}$ depth and $1 \mathrm{~mm}$ width (double-channel chip): one channel for venipuncture blood and the other for fingerprick capillary blood. It has two inlets and a single shared outlet. The chip patterns were drawn using a CAD software (Autodesk, AutoCAD 2016) and fabricated by CNC machining.

For aggregation monitoring, channels with smaller depths are needed because of the high population and small size of red blood cells (around 5-7 $\mu \mathrm{m}$ ) that make their visualization challenging in deep channels. CNC-machined PMMA chips are not suitable for monitoring of red blood cells for two reasons. First, CNC-machining leaves a rough surface reducing the transparency of the channel. Second, thicknesses of the commercially available PMMA layers are not uniform, leading to chip-to-chip variation in microfabrication. Therefore, a shallow PDMS microfluidic chip was designed for visualization of red blood cell aggregation (Cai and Neyer 2010; Viero et al. 2012). The following equation should be satisfied to apply the same shear rates on cells in different channel segments when the same pressure is applied:

$w_{1} h_{1}^{2}=w_{2} h_{2}^{2}$

where $w$ is the channel width and $h$ is the channel height (Isiksacan et al. 2016a). Therefore, the width of the PDMS chip was defined according to the width $(1 \mathrm{~mm})$ and the height $(1 \mathrm{~mm})$ of the channel of the PMMA chip. The width was set to $150 \mu \mathrm{m}$ for better cell visualization. The PDMS chip therefore consisted of a $150 \mu \mathrm{m}$ deep,
$44.5 \mathrm{~mm}$ wide, and $50 \mathrm{~mm}$ long channel. The PDMS chip was fabricated based on a modified version of a rapid fabrication method recently reported (Isiksacan et al. 2016b). Firstly, the patterns were drawn using a CAD software (Autodesk, AutoCAD 2016). Then, $150 \mu \mathrm{m}$ thick adhesive tape was applied on a PMMA substrate, and the tape was laser-ablated using a $30 \mathrm{~W} \mathrm{CO}_{2}$ laser (Epilog, Zing) to obtain the desired patterns. After the ablation, the offchannel regions were peeled off the substrate finalizing the mold. PDMS (Sylgard 184, Dow Corning), prepared at 10:1 ratio and debubbled, was poured onto the mold, and the mold was placed on a hotplate at $40{ }^{\circ} \mathrm{C}$ for $12 \mathrm{~h}$ for PDMS curing. Then, the cured PDMS was removed from the mold, inlet and outlet holes were punched, and the PDMS was bonded to a glass slide using $\mathrm{O}_{2}$ plasma.

\subsection{Measurement system}

We used the microfluidic system that is schematically shown in Fig. 1 (Isiksacan et al. 2016a). It consists of a PMMA microfluidic chip and an opto-electro-mechanical analyzer. In the analyzer, a solenoid pinch valve (EW-98302-06) was used in order to shake the blood sample. This valve generates continuous back and forth motion of the blood sample in the channel, and thus disaggregates the red blood cells. For the quantification of the interaction between the incident light and cells, which forms the basis of the working principle of the measurement system, an optical system was developed. The analyzer contains a light-emitting diode (VSMG2700, Vishay) in the near-infrared (NIR) band (wavelength $=830 \mathrm{~nm}$ ) and a photodetector (VEMT4700, Vishay), former at the top and latter at the bottom of the chip, respectively. Using NIR light, the optical transmission of hemoglobin is minimized, and thus higher transmission signal is obtained (Uyuklu et al. 2011). For comparison of ESR results

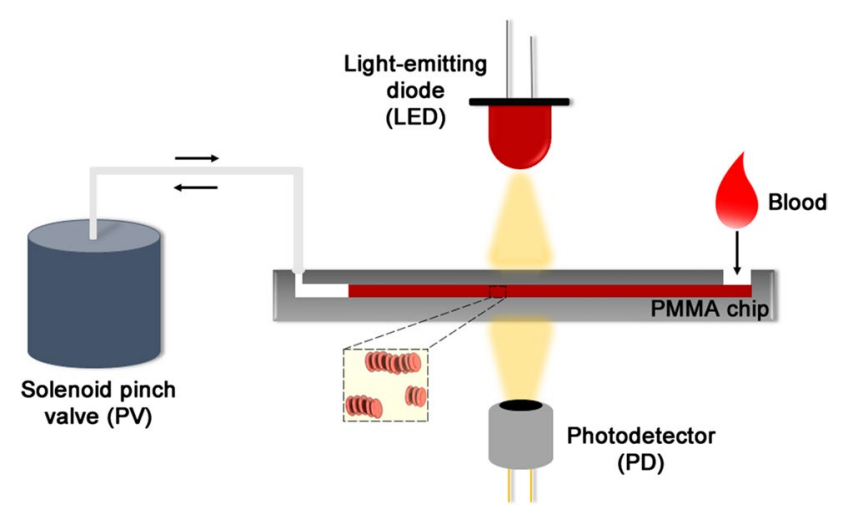

Fig. 1 Schematic representation of the microfluidic system used for ESR measurement. Solenoid pinch valve is used to disaggregate the cells after blood sample is filled in the PMMA chip. Photodetector measures the transmitted optical signal through the channel 
from venous and capillary blood samples, another analyzer was developed which contained two light-emitting diodes and two photodetectors. Each set of light source and photodetector was aligned with one of the parallel channels of the PMMA chip. The test cycle starts off by filling $40 \mu \mathrm{l}$ blood sample into the inlet port of a channel. Red blood cells cluster immediately after they are introduced to the microfluidic chip. Following the sample injection into the channel through the inlet port, the pinch valve operates for $15 \mathrm{~s}$ to disaggregate the cell clusters. When the cells completely disaggregate, the intensity of the transmitted optical signal measured by the photodetector is at the lowest value. As the cells start reaggregating, intensity increases until the cells completely aggregate, after which the intensity stays constant.

\section{Results and discussion}

\subsection{ESR measurement using dextran-mediated blood}

As discussed in the materials section, five blood samples with different dextran concentrations were prepared: $0,5,10,15$, and $20 \mathrm{mg} / \mathrm{ml}$. Since red blood cells were suspended in PBS, all the macromolecules affecting the aggregation dynamics were out of play. Only consideration was the $70 \mathrm{kDa}$ dextran that was known to alter the aggregation dynamics, by either inhibiting or enhancing aggregation depending on the concentration (Neu et al. 2008). $40 \mu \mathrm{l}$ of each sample was pipetted into a single-channel PMMA chip, and the optical transmission signal was collected for $150 \mathrm{~s}$. Each sample was tested four times. Since the four signal profiles were almost the same, only one signal for each sample was presented in Fig. 2a. For the optical transmission signals, three sedimentation indices $\left(\mathrm{SI}_{1}\right.$, $\mathrm{SI}_{2}$, and $\mathrm{SI}_{3}$ ) are calculated for the quantification of aggregation (Fig. 2b) (Baskurt et al. 2011a). SI $_{1}$ is calculated by taking the ratio of the area under the signal curve (AUC) to the total area of the signal (AUC $+\mathrm{AAC}$ ). $\mathrm{SI}_{2}$ is the amplitude of the optical signal intensity, and $\mathrm{SI}_{3}$ is the time when the intensity reaches half of the maximum value. For a set of sedimentation index values, each value is also divided by the maximum value of the set. These indices were calculated at 30th, 60th, 90th, and 120th s of the optical transmission data. Figure $2 \mathrm{c}$ shows the calculated $\mathrm{SI}_{1}$ values at different dextran concentrations and at different analysis times. Similarly, Fig. 2d, e illustrate the $\mathrm{SI}_{2}$ and $\mathrm{SI}_{3}$ values, a

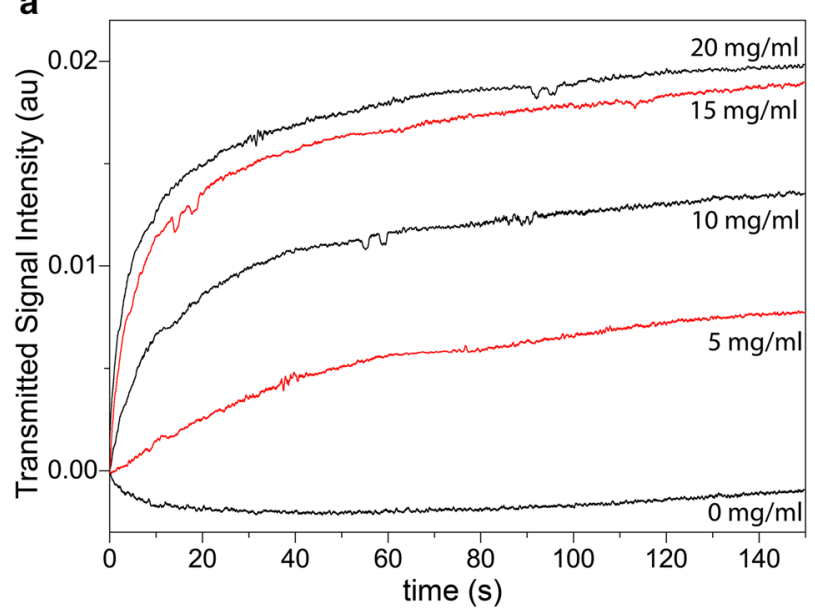

b

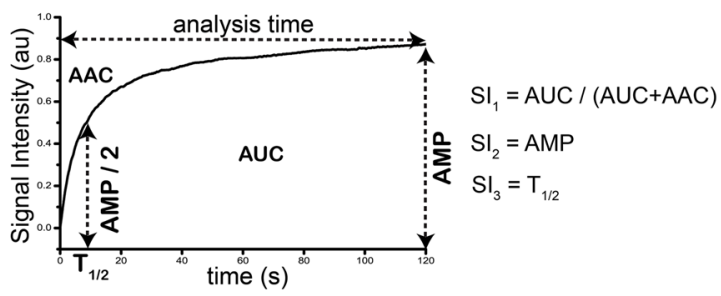

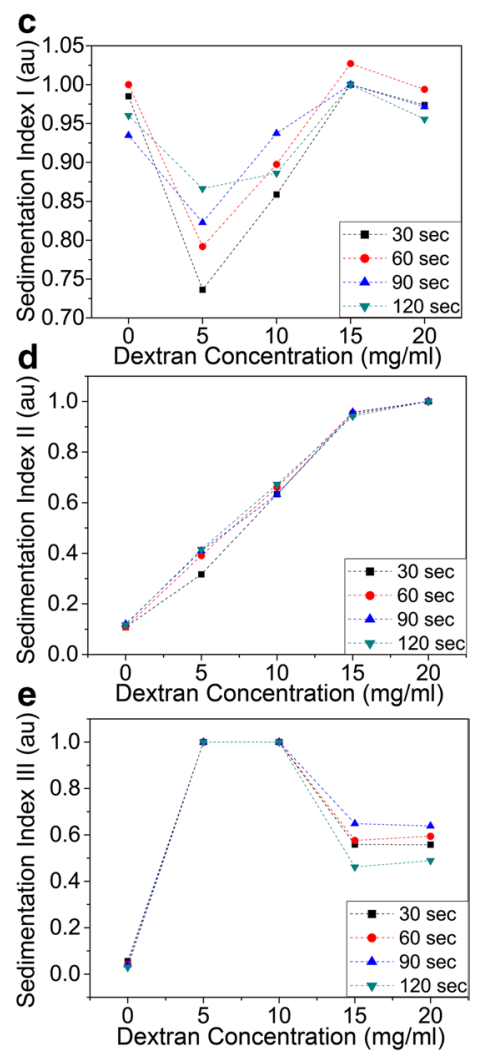

Fig. 2 Blood samples with different dextran concentrations tested with the microfluidic system. a Transmitted signal intensities of five samples were measured using the system. The signals are offset to zero initial intensity. b Schematic drawing of a representative trans- mission signal and the sedimentation indices calculated from the signal. c SI ${ }_{1}, \mathbf{d ~ S I}{ }_{2}$, and $\mathbf{e} \mathrm{SI}_{3}$ at different dextran concentrations and analysis times 
respectively. According to these results, it is evident that amplitude $\left(\mathrm{SI}_{2}\right)$ is the only index that increases almost linearly with the dextran concentration. In addition, at a given dextran concentration, $\mathrm{SI}_{2}$ does not vary significantly as a function of analysis time. Therefore, $\mathrm{SI}_{2}$ is accepted as the most legitimate index for the quantification of aggregation. Additionally, Fig. 2d confirms the bridging hypothesis. (1) An increase in the concentration of dextran increases the amplitude of the transmitted signal intensity. (2) The increasing signal intensity implies the formation of larger aggregates. Therefore, we conclude that dextran macromolecules are adsorbed onto the cell surfaces to enhance aggregation.

As the optical measurements were carried out using the microfluidic system, $75 \mu$ from each of the five blood samples was taken into a hematocrit tube (inner diameter: $1 \mathrm{~mm}$, length: $75 \mathrm{~mm}$ ). Then, the tubes were placed vertically on a stationary rack to measure the sedimentation rates of the red blood cells for each sample using the conventional method (Fig. 3a). The five hematocrit tubes containing the samples of various concentrations were observed for a total of $4 \mathrm{~h}$. At the end of the $1 \mathrm{st}, 2 \mathrm{nd}, 3 \mathrm{rd}$, and 4 th $\mathrm{h}$, pictures of the tubes were taken, and ESR was calculated from these pictures. These values were used to compare the conventional measurement results and the microfluidic measurement results obtained using $\mathrm{SI}_{2}$ at various analysis times (Fig. 3b). According to Fig. 3b, the correlation between the conventional measurement results and the microfluidic measurement results is above $80 \%$ for all the analysis times of the optical signal (30th, 60th, 90th, and 120th s) and at any reading time of the ESR from the hematocrit tubes (1st, 2nd, 3rd, and 4th h). Variations among correlation coefficient values for different optical signal analysis times and different ESR reading times are due to several internal factors including sample viscosity, ambient temperature, and cell deformability. These factors might have different effects on cell sedimentation (conventional measurement) and cell aggregation (microfluidic measurement). Specifically, Fig. 3c gives correlation coefficient $\left(\% R^{2}\right)$ of $87 \%$ for 30 th $\mathrm{s}$ analysis time and $1 \mathrm{st} \mathrm{h}$ conventional ESR reading time using linear regression, and Fig. 3d gives correlation coefficient $\left(\% R^{2}\right)$ of $98.5 \%$ for 120th $\mathrm{s}$ analysis time and 4th $\mathrm{h}$ conventional ESR reading time using linear regression. This confirms that the sedimentation rate of red blood cells obtained from the conventional method and the aggregation rate of red blood cells obtained from the microfluidic method have a direct relationship, proving the validity of the developed system for
Fig. 3 Comparison of the conventional ESR measurement and the microfluidic measurement. a Schematic representation of the conventional ESR measurement with hematocrit tubes. b Goodness of fit of the linear regression curve for conventional measurement result versus microfluidic measurement result at different signal analysis times and different hematocrit tube reading times. c Comparison of the conventional ESR measurement read after $1 \mathrm{~h}$ of sample introduction and $\mathrm{SI}_{2}$ of the optical transmission signal calculated at 30th $\mathrm{s}$ analysis time. $\mathbf{d}$ Comparison of the conventional ESR measurement read after $4 \mathrm{~h}$ of sample introduction and $\mathrm{SI}_{2}$ of the optical transmission signal calculated at 120 th s analysis time

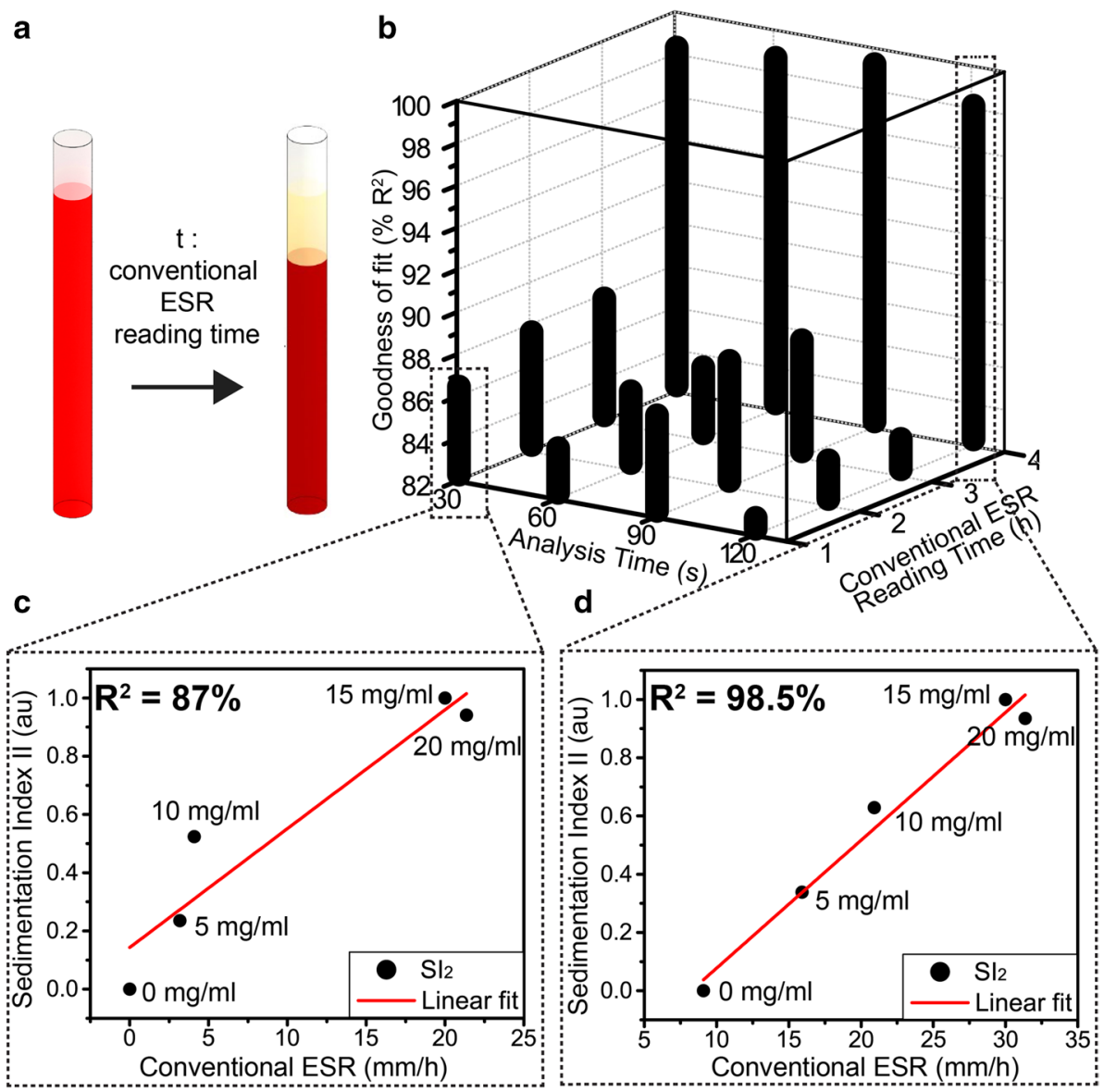


ESR measurement. From Fig. 3b, it is also clear that the analysis time of $90 \mathrm{~s}$ gives the highest correlation. Therefore, this analysis time was used at further studies.

\subsection{Effect of measurement conditions}

For point-of-care applications, the lab-on-a-chip systems should endure off-laboratory use by non-specialists (Chin et al. 2007, 2012). Normally, similar diagnostic devices operate on a laboratory bench or a desk. On the other hand, hand-held devices should be robust enough to handle a variety of the use scenarios during the operation (Chin et al. 2012; Sia and Kricka 2008; Yager et al. 2008). Therefore, we investigated the performance of the microfluidic system when the device orientation was varied, where the extreme condition was the vertical device orientation. In addition, such a comparison is critical to verify that the underlying effect, which yields the optical signal demonstrated in Fig. 2a, is the aggregation of red blood cells, rather than their sedimentation.

For these experiments, we used the PBS-suspended and $10 \mathrm{mg}$ dextran-added blood sample (T3 tube). The sample was tested 5 times in each of the two device orientations, horizontal and vertical, using single-channel chips. The optical transmission signals obtained from these 10 tests were analyzed to calculate $\mathrm{SI}_{2}$ at 90 th $\mathrm{s}$ for each test. Following it, the mean and standard deviation of the results at the horizontal and vertical device orientation were calculated. When compared, the mean values of 0.98 (horizontal) and 0.99 (vertical) show that the results are independent of device orientation. The low standard deviation values in both cases (horizontal S.D. $=0.01$, vertical S.D. $=0.001$ ) demonstrate the high repeatability of the rapid ESR measurement. These tests also verify that the developed system investigates the aggregation dynamics within the channel rather than the sedimentation effect of the cells that would otherwise have had different effects at the two device orientations.

For devices, which utilize optical measurement/detection mechanism, vibrations can interfere with the measurement results by increasing the baseline noise level (Mogensen and Kutter 2009; Myers and Lee 2008). Therefore, we also investigated the measurement performance of the system when the device is mechanically disturbed. Disturbance is created by harshly hitting with a plastic hammer at a region on the desk that was $20 \mathrm{~cm}$ far from the system. A total of six tests were performed. Each disturbance generated a mean instant force of $157 \mathrm{~N}( \pm 20 \mathrm{~N})$ at the hitting region. Figure 4 shows the intensities of the transmission signals obtained from the tests using $10 \mathrm{mg}$ dextran-added sample. The black squares in the plots denote the times at which the disturbance was created during the tests. After the tests, the $\mathrm{SI}_{2}$ at 90th $\mathrm{s}$ was calculated from the optical signals for each test. The mean of the $\mathrm{SI}_{2}$ values of the six tests (mean $=0.91)$ as well as the standard deviation of the test results (S.D. $=0.046)$ were calculated. The standard deviation is in an acceptable range, implying that the mechanical disturbance did not affect the test performance.

\subsection{Effect of shear rate on red blood cell disaggregation}

One of the major issues of the presented microfluidic ESR system and the conventional Westergren assay is the dependence of the results to the initial condition of the sample, which is assumed to be complete disaggregation of red blood cells. For the conventional assay, it is expected that operator disaggregates the blood sample by repetitive gentle upside down motion of the blood tube. Our system frees the user from this constraint by performing a sample shaking action for $15 \mathrm{~s}$ before initiating the optical measurement. However, complete disaggregation of the sample depends on the microfluidic channel design, sample amount, and the mechanical unit used for disaggregation. In this section, we evaluate the performance of the pinch valve in achieving the complete disaggregation state in order to develop a foolproof sample conditioning for our system.

Blood is a non-Newtonian fluid demonstrating shearthinning behavior (Anand and Rajagopal 2004; Cokelet 1987). Different models, such as Cross (Cross 1965), Walburn-Schnock (Walburn and Schneck 1976), Carreau (Carreau et al. 1968; Gambaruto et al. 2011), have been developed to explain the non-linear shear rate and shear stress relation for non-Newtonian fluids. Among all, the CarreauGambaruto model has gained more attention due to the higher agreement between computational and experimental

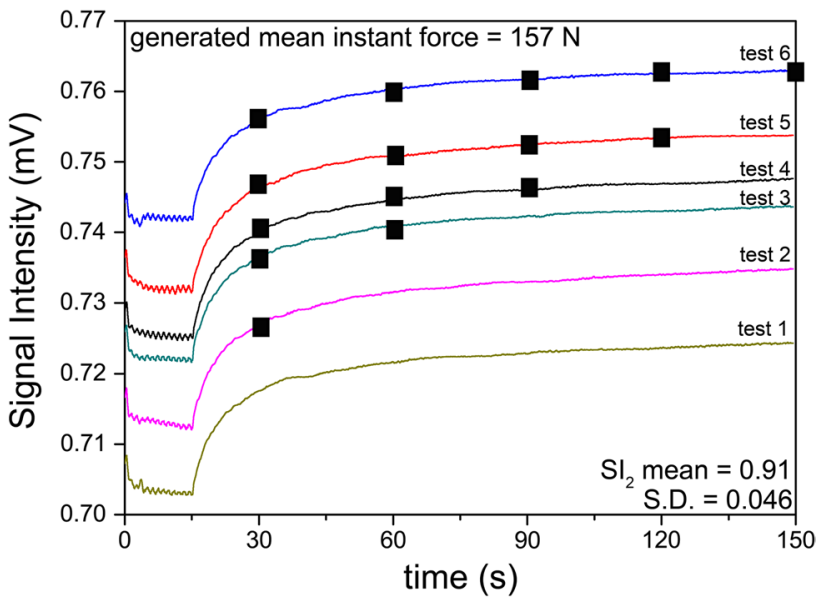

Fig. 4 Investigation of the effect of vibration on the optical measurements. Six tests were performed using the same blood sample. Black squares denote the times for external disturbance of $157 \mathrm{~N}( \pm 20 \mathrm{~N})$. Mean and standard deviation calculated from the $\mathrm{SI}_{2}$ values at the 90th $\mathrm{s}$ for each signal were given 
results for blood (Mimouni 2016). According to the Carreau model, the viscosity of a non-Newtonian fluid can be given as:

$\mu=\mu_{\mathrm{inf}}+\left(\mu_{0}-\mu_{\mathrm{inf}}\right)\left[1+(\lambda \dot{\gamma})^{2}\right]^{(n-1) / 2}$

where $\mu$ is viscosity, $\dot{\gamma}$ is shear rate, $\mu_{0}$ and $\mu_{\text {inf }}$ are the zero and infinite shear rate limit viscosities, $\lambda$ is the relaxation time constant, and $n$ is the power law index. For blood, the constants were previously reported as $\mu_{0}=0.0456$ Pa.s, $\mu_{\text {inf }}=0.0032$ Pa.s, $\lambda=10.03 \mathrm{~s}$, and $n=0.344$ (Gambaruto et al. 2011).

Due to its low cost and ease of integration, we used a pinch valve in our system to achieve complete red blood cell disaggregation before the onset of the optical measurement. We aimed to see the effect of the pinch valve on the disaggregation of red blood cells using the CarreauGambaruto model. A finite element modeling software, COMSOL 5.0, was employed to study this effect. First, the PMMA channel with $1 \mathrm{~mm}$ width, $1 \mathrm{~mm}$ depth, and $50 \mathrm{~mm}$ length was drawn as shown in Fig. 5a. The laminar flow regime was applied with momentum balance and continuity equations using the shear parameters given in the Carreau-Gambaruto model for blood. There are several pinch valves that are commercially available. As an example, we compared two pinch valves designed for different tubing dimensions for their disaggregation performance: EW-98302-06 (Cole-Parmer) and EW-98302-00 (Cole-Parmer). When these pinch valves were employed in the microfluidic system, the flow rates of blood in the channel was measured as 5 and $0.6 \mathrm{~mm}^{3} / \mathrm{s}$, respectively. These flow rates were applied to the software for the inlets, and atmospheric pressure was used as the outlet boundary condition. The fluid was assumed to be fully developed. No slip boundary condition was used for the walls, and the fluid was assumed to be incompressible. Fine meshes were created that gave 1,002,047 elements. Mesh types for cross sections and along the channels were free triangular and swept, respectively. Figure 5a, $\mathrm{b}$ give the shear rate distributions along the channel for EW-98302-06 (PV-1) and EW-98302-00 (PV-2) pinch valves, respectively. To further investigate the shear rates formed at different points in the channel, center lines were drawn along one of the identical side of the channel surfaces and the channel center. As shown in Fig. 5c, for the channel center line, PV-1 creates shear rates that vary between 2.5 and $4.5 \mathrm{~s}^{-1}$, and PV-2 creates shear rate around $0.1 \mathrm{~s}^{-1}$ along the channel. Along the channel surfaces, shear rates applied to the cells by PV-1 and PV-2 are around 50 and $6 \mathrm{~s}^{-1}$, respectively as shown in
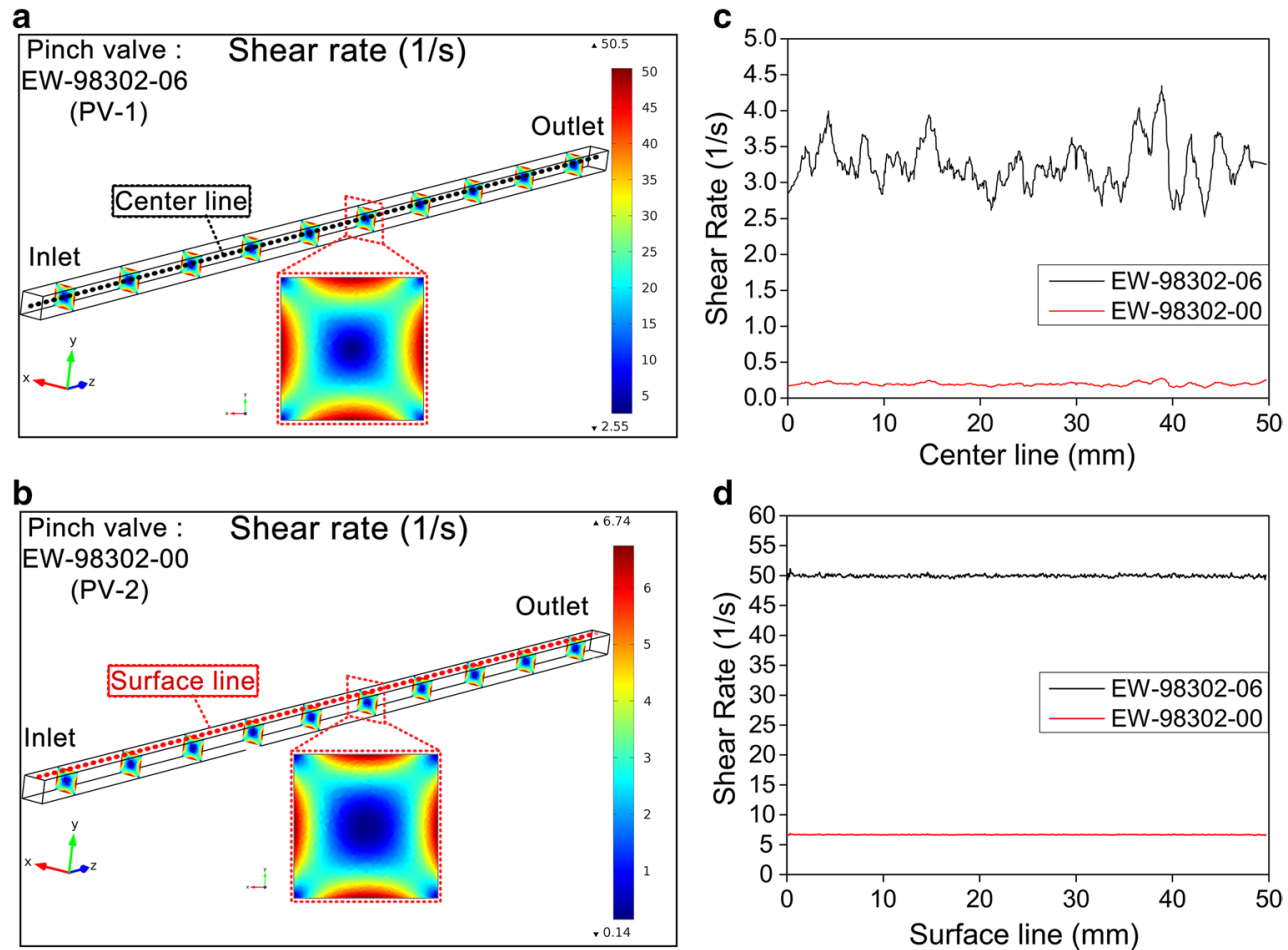

Fig. 5 Finite element model results showing the shear rate distributions applied to red blood cells in the channels for different pinch valves. a Shear rate distribution when PV-1 pinch valve was employed. b Shear rate distribution when PV-2 pinch valve was employed. c Shear rate along the center line passing through the center of the channel. d Shear rate along the line passing through the center of bottom/top/left/right surface, respectively 
Fig. 5d. It was previously demonstrated that shear rates below $1 \mathrm{~s}^{-1}$ is not sufficient for red blood cell disaggregation. An effective shear rate range for disaggregation was reported to be $1 \mathrm{~s}^{-1}<\dot{\gamma}<100 \mathrm{~s}^{-1}$ (Mimouni 2016). These results suggest that PV-2 fails to provide the threshold shear rate required for red blood cell disaggregation, whereas $\mathrm{PV}-1$ provides the required shear rate both at the channel surface and the center.

To experimentally verify our computational results, two PDMS chips were fabricated for microscopic observation: one for PV-1 and the other for PV-2. We pipetted the dextran-added dilute blood samples (see Materials section) into the chips. PV-1 and PV-2 were connected to the outlets of the chips. The pinch valves were operated for $15 \mathrm{~s}$, and the cells were observed under microscope using $40 \times$ microscope objective (Axio Vert.A1, Zeiss) immediately after stopping the pinch valve action. As shown in Fig. 6a, b, there is significant difference between the two cases. PV-1 can achieve complete disaggregation, whereas PV-2 falls short in applying enough shear rate to disaggregate the cells resulting in large rouleaux after the pump stops as seen in Fig. 6b. It is important to note that Fig. 6a shows some small stacks of red blood cells which formed after the cease of the valve's operation, confirming the rapid timescale of aggregation process reported in the literature (Baskurt et al. 2011a).

\subsection{ESR measurement using whole blood}

The microfluidic platforms that investigate human blood for either clinical or research purposes generally use venous blood obtained by venipuncture. However, most point-ofcare devices are used by non-technical personnel and work with fingerprick blood that is much easier to collect. It is a concern that fingerprick capillary blood may lead to erroneous results due to the fact that it contains higher amount of interstitial fluid and cell debris compared to venipuncture blood. In order to verify the potential of our microfluidic system as a point-of-care ESR device, we compared the results obtained from venous and capillary blood samples. For this purpose, we developed a PMMA microfluidic chip with two parallel channels (channel-A and channelB) with individual inlets and a shared outlet (Fig. 7b, c). The chip was fabricated by CNC-machining. ChannelA was reserved for venipuncture blood, while channel-B was reserved for fingerprick blood. In order to prevent the coagulation of fingerprick blood, channel-B was washed for $20 \mathrm{~s}$ with $10 \mu \mathrm{l}$ of EDTA solution $(1.5 \mathrm{mg} / \mathrm{ml})$ obtained from a Vacutainer blood collection tube (Becton-Dickinson). The EDTA solution was simply ran through channel$\mathrm{B}$, and then the chip was left to dry for $30 \mathrm{~min}$ at room temperature. The leftover EDTA residue on the channel walls was enough to prevent coagulation of capillary blood. We have also improved the analyzer to have two sets of LEDphotodetector pairs, aligned with one of the parallel channels. Then, the following experiments were performed:

1. Whole blood of a donor was drawn by venipuncture into a vacuum tube containing EDTA $(1.5 \mathrm{mg} / \mathrm{ml})$. It was tested using the single-channel chip and the analyzer with one LED-PD pair. $40 \mu \mathrm{l}$ blood was pipetted from the tube into the channel. Three repetitive tests were performed using different chips. The optical transmission signals obtained from these three tests were analyzed to calculate the $\mathrm{SI}_{2}$ at 90 th $\mathrm{s}$ for each test. Figure 7 a shows the test results and the schematic representation of the system.

2. The venous blood sample used in part-a was tested using the double-channel chip and the analyzer containing two LED-PD pairs. $40 \mu \mathrm{l}$ blood was pipetted from the tube into channel-A. Three repetitive tests were performed using different chips. The same steps were
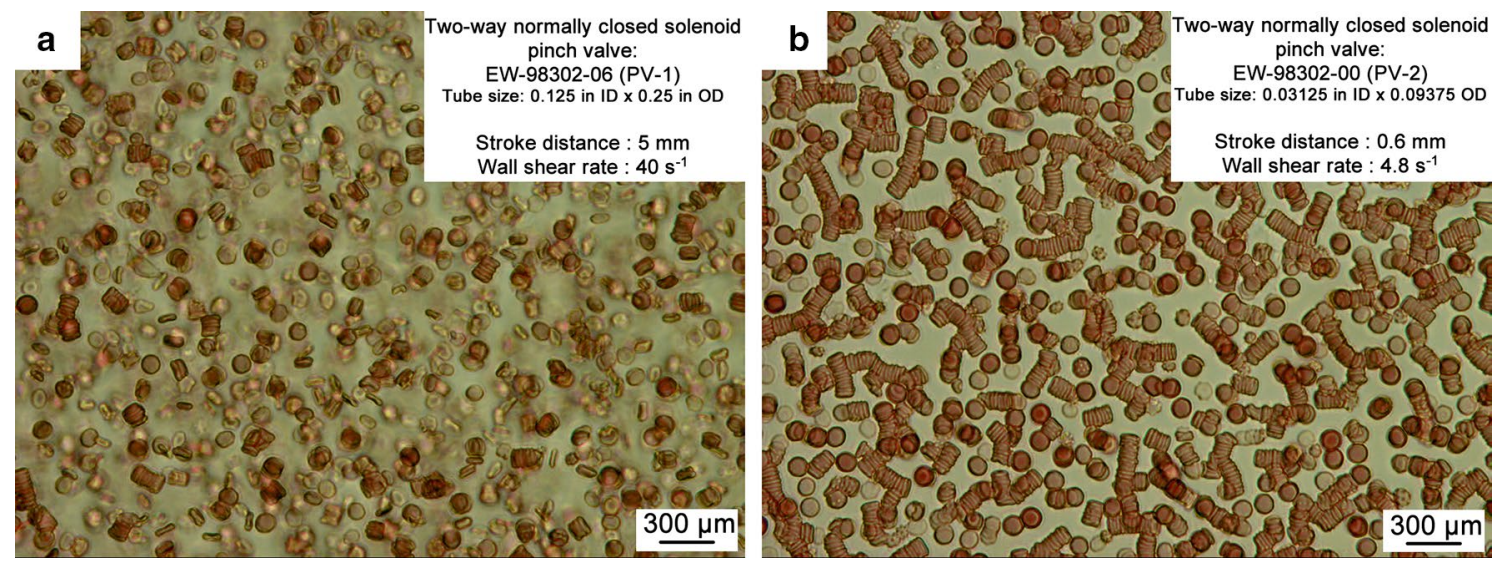

Fig. 6 Visual observation of red blood cells just after the pinch valves stopped operating. a PV-1 was employed, and cells were disaggregated. b PV-2 was employed, and disaggregation was not achieved 


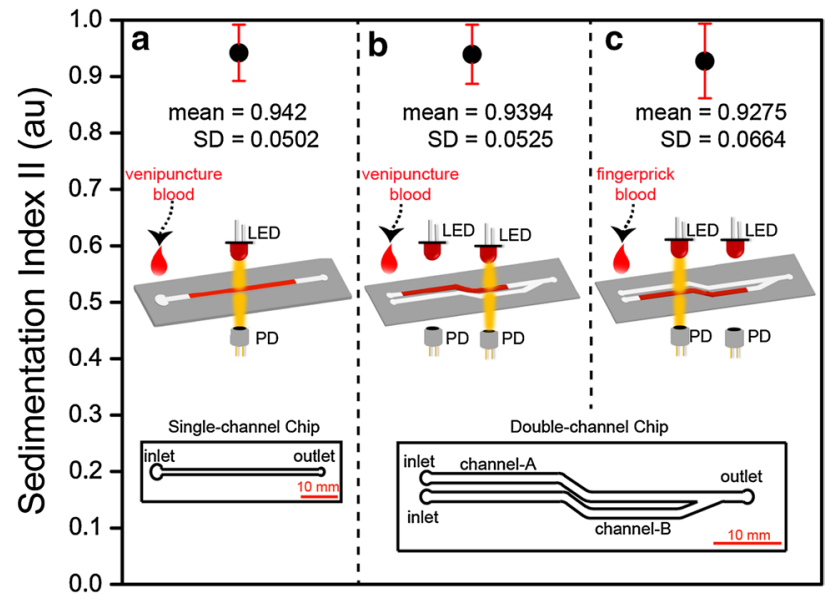

Fig. 7 ESR measurement of venipuncture versus fingerprick whole blood using different chips and analyzers. Three repetitive tests were performed and mean $\mathrm{SI}_{2}$ was calculated for each part. $a$ Single-channel chip and the analyzer with one LED-PD pair were employed for venipuncture blood. $b$ Double-channel chip and the analyzer with two LED-PD pairs were employed for venipuncture blood. Sample was introduced into channel-A. $c$ Double-channel chip and the analyzer with two LED-PD pairs were employed for fingerprick blood. Sample was introduced into channel-B that was heparinized before test

followed as given in part-a. Figure $7 \mathrm{~b}$ shows the test results and the schematic representation of the system.

3. $40 \mu \mathrm{l}$ fingerprick capillary blood was obtained from the same donor and introduced into channel-B of the double-channel chip which was coated with EDTA. Three repetitive tests were performed using different chips. The same steps were followed as given in part-a and part-b. Figure $7 \mathrm{c}$ shows the test results and the schematic representation of the system.

The results of three repetitive tests for each part were consistent as presented in Fig. 7. The mean values of $\mathrm{SI}_{2}$ calculated at 90th $\mathrm{s}$ of the optical signals are 0.9420 (Fig. 7a), 0.9394 (Fig. 7b), and 0.9275 (Fig. 7c). Also, the standard deviation for each part is acceptable. The mean values and the standard deviations are in good agreement for all three cases despite slightly higher S.D. for fingerprick blood samples. It is therefore apparent that the presented system can be used as a rapid ESR measurement system that can work as a point-of-care system using fingerprick blood sample as well as a laboratory unit that operates using whole blood collected in anticoagulated blood tubes.

\section{Conclusion}

We demonstrated the feasibility of a microfluidic platform for point-of-care measurement of erythrocyte sedimentation rate utilizing the aggregation dynamics of red blood cells within $40 \mu$ blood samples. Dextranmediated blood samples with different dextran concentration were prepared, which showed varying red blood cell aggregability properties. A dextran concentration interval was found where red blood cell aggregation and correspondingly sedimentation rate increase linearly with the increasing concentration. We showed that the orientation of the device does not alter the test results, validating the use of the microfluidic system for hand-held use by non-professionals. Additionally, mechanical disturbances during the test were shown not to affect the optical signals obtained from the system. Also, two different pinch valves were integrated to the system, and their performances for forming the required shear rate allowing red blood cell disaggregation were characterized computationally and experimentally. Finite element modelling was employed to find the shear rates on cells at different locations along the channel. Afterwards, the pinch valves were integrated to the system, and the stronger valve was experimentally shown to successfully disaggregate the cells. Finally, with a novel chip design and addition of another LED-photodetector pair to the analyzer, the system was shown to perform ESR measurement from both venipuncture and fingerprick whole blood. This feature enables the applicability of the microfluidic ESR system at physician office laboratory, central laboratories, and at the point-of-care. In addition, the system features the ease-of-use, portability, and durability required for rapid ESR measurements at resource-poor settings.

Acknowledgements The authors acknowledge support from The Scientific and Technological Research Council of Turkey (TUBITAK project no. 213S127).

\section{References}

Anand M, Rajagopal K (2004) A shear-thinning viscoelastic fluid model for describing the flow of blood. Int J Cardiovasc Med Sci 4:59-68

Andresdottir MB, Sigfusson N, Sigvaldason H, Gudnason V (2003) Erythrocyte sedimentation rate, an independent predictor of coronary heart disease in men and women the Reykjavik study. Am J Epidemiol 158:844-851

Andrews DA, Low PS (1999) Role of red blood cells in thrombosis. Curr Opin Hematol 6:76

Armstrong JK, Meiselman HJ, Wenby RB, Fisher TC (2001) Modulation of red blood cell aggregation and blood viscosity by the covalent attachment of Pluronic copolymers. Biorheology 38:239-247

Asakura S, Oosawa F (1954) On interaction between two bodies immersed in a solution of macromolecules. J Chem Phys 22:1255-1256

Barshtein G, Tamir I, Yedgar S (1998) Red blood cell rouleaux formation in dextran solution: dependence on polymer conformation. Eur Biophys J 27:177-181 
Baskurt OK, Meiselman HJ (2013) Erythrocyte aggregation: basic aspects and clinical importance. Clin Hemorheol Microcirc 53:23-37

Baskurt OK, Meiselman H, Kayar E (1998) Measurement of red blood cell aggregation in a "plate-plate" shearing system by analysis of light transmission. Clin Hemorheol Microcirc 19:307-314

Baskurt OK, Uyuklu M, Hardeman MR, Meiselman HJ (2009) Photometric measurements of red blood cell aggregation: light transmission versus light reflectance. J Biomed Opt 14:054044

Baskurt OK, Uyuklu M, Meiselman HJ (2010) Time course of electrical impedance during red blood cell aggregation in a glass tube: comparison with light transmittance. IEEE Trans Biomed Eng 57:969-978

Baskurt O, Neu B, Meiselman HJ (2011a) Red blood cell aggregation. CRC Press, Boca Raton

Baskurt OK, Uyuklu M, Ozdem S, Meiselman HJ (2011b) Measurement of red blood cell aggregation in disposable capillary tubes. Clin Hemorheol Microcirc 47:295-305

Bishop JJ, Popel AS, Intaglietta M, Johnson PC (2001) Rheological effects of red blood cell aggregation in the venous network: a review of recent studies. Biorheology 38:263-274

Bull BS, Brailsford JD (1972) The zeta sedimentation ratio. Blood 40:550-559

Cai D, Neyer A (2010) Cost-effective and reliable sealing method for PDMS (PolyDiMethylSiloxane)-based microfluidic devices with various substrates. Microfluid Nanofluidics 9:855-864

Carreau PJ, MacDonald IF, Bird RB (1968) A nonlinear viscoelastic model for polymer solutions and melts-II. Chem Eng Sci 23:901-911

Chien S (1975) Biophysical behavior of red cells in suspensions. Red Blood Cell 2:1031-1133

Chien S, Jan KM (1973) Red cell aggregation by macromolecules: roles of surface adsorption and electrostatic repulsion. J Supramol Struct 1:385-409

Chien S, Usami S, Dellenback RJ, Gregersen MI, Nanninga LB, Guest MM (1967) Blood viscosity: influence of erythrocyte aggregation. Science 157:829-831

Chin CD, Linder V, Sia SK (2007) Lab-on-a-chip devices for global health: past studies and future opportunities. Lab Chip 7:41-57

Chin CD, Linder V, Sia SK (2012) Commercialization of microfluidic point-of-care diagnostic devices. Lab Chip 12:2118-2134

Cokelet G (1987) The rheology and tube flow of blood. In: Skalak $\mathrm{R}$, Chien S (eds) Handbook of bioengineering, vol 14. McGrawHill, New York, pp 14.1-14.7

Cross MM (1965) Rheology of non-Newtonian fluids: a new flow equation for pseudoplastic systems. J Colloid Sci 20:417-437

Deng L, Barbenel J, Lowe G (1993) Influence of hematocrit on erythrocyte aggregation kinetics for suspensions of red blood cells in autologous plasma. Biorheology 31:193-205

Dima A, Opris D, Jurcut C, Baicus C (2016) Is there still a place for erythrocyte sedimentation rate and C-reactive protein in systemic lupus erythematosus? Lupus 25:1173-1179

Gambaruto AM, Janela J, Moura A, Sequeira A (2011) Sensitivity of hemodynamics in a patient specific cerebral aneurysm to vascular geometry and blood rheology. Math Biosci Eng MBE 8:409-423

Gillum RF, Mussolino ME, Makuc DM (1995) Erythrocyte sedimentation rate and coronary heart disease: the NHANES I epidemiologic follow-up study. J Clin Epidemiol 48:353-361

Gilmour D, Sykes A (1951) Westergren and Wintrobe methods of estimating ESR compared. Br Med J 2:1496

Hardwicke J, Squire J (1952) The basis of the erythrocyte sedimentation rate. Clin Sci 11:333-355

Hong T-F, Ju W-J, Wu M-C, Tai C-H, Tsai C-H, Fu L-M (2010) Rapid prototyping of PMMA microfluidic chips utilizing a $\mathrm{CO}_{2}$ laser. Microfluid Nanofluidics 9:1125-1133
Hysi E, Saha RK, Kolios MC (2012) On the use of photoacoustics to detect red blood cell aggregation. Biomed Opt Express 3:2326-2338

Isiksacan Z, Erel O, Elbuken C (2016a) A portable microfluidic system for rapid measurement of the erythrocyte sedimentation rate. Lab Chip 16:4682-4690

Isiksacan Z, Guler MT, Aydogdu B, Bilican I, Elbuken C (2016b) Rapid fabrication of microfluidic PDMS devices from reusable PDMS molds using laser ablation. J Micromech Microeng 26:035008

Jan K-M, Chien S (1973) Role of surface electric charge in red blood cell interactions. J Gen Physiol 61:638-654

Jiang H, Weng X, Li D (2011) Microfluidic whole-blood immunoassays. Microfluid Nanofluidics 10:941-964

Johansson JE, Sigurdsson T, Holmberg L, Bergstrom R (1992) Erythrocyte sedimentation rate as a tumor marker in human prostatic cancer. An analysis of prognostic factors in 300 population-based consecutive cases. Cancer 70:1556-1563

Li C, Liu C, Xu Z, Li J (2012) A power-free deposited microbead plug-based microfluidic chip for whole-blood immunoassay. Microfluid Nanofluidics 12:829-834

Lim H-J, Lee Y-J, Nam J-H, Chung S, Shin S (2010) Temperaturedependent threshold shear stress of red blood cell aggregation. J Biomech 43:546-550

Lominadze D, Joshua IG, Schuschke DA (1998) Increased erythrocyte aggregation in spontaneously hypertensive rats. Am J Hypertens 11:784-789

Manage DP, Morrissey YC, Stickel AJ, Lauzon J, Atrazhev A, Acker JP, Pilarski LM (2011) On-chip PCR amplification of genomic and viral templates in unprocessed whole blood. Microfluid Nanofluidics 10:697-702

Mehri R, Mavriplis C, Fenech M (2014) Design of a microfluidic system for red blood cell aggregation investigation. J Biomech Eng 136:064501

Merrill EW (1969) Rheology of blood. Physiol Rev 49:863-888

Mimouni Z (2016) The rheological behavior of human bloodcomparison of two models open. J Biophys 6:29

Mogensen KB, Kutter JP (2009) Optical detection in microfluidic systems. Electrophoresis 30:S92-S100

Myers FB, Lee LP (2008) Innovations in optical microfluidic technologies for point-of-care diagnostics. Lab Chip 8:2015-2031

Nash GB, Meiselman HJ (1983) Effects of dextran and polyvinylpyrrolidone on red cell geometry and membrane elasticity. Ann N Y Acad Sci 416:255-262

Neu B, Meiselman HJ (2007) Red blood cell aggregation. In: Baskurt OK, Hardeman MR, Rampling MW, Meiselman HJ (eds) Handbook of hemorheology and hemodynamics, vol 69. IOS Press, Amsterdam, pp 114-136

Neu B, Wenby R, Meiselman HJ (2008) Effects of dextran molecular weight on red blood cell aggregation. Biophys $\mathrm{J}$ 95:3059-3065

Plebani M, De Toni S, Sanzari MC, Bernardi D, Stockreiter E (1998) The TEST 1 automated system: a new method for measuring the erythrocyte sedimentation rate. Am J Clin Pathol 110:334-340

Rampling M, Meiselman H, Neu B, Baskurt O (2004) Influence of cell-specific factors on red blood cell aggregation. Biorheology 41:91-112

Shin S, Nam J-H, Hou J-X, Suh J-S (2009) A transient, microfluidic approach to the investigation of erythrocyte aggregation: the threshold shear-stress for erythrocyte disaggregation. Clin Hemorheol Microcirc 42:117-125

Sia SK, Kricka LJ (2008) Microfluidics and point-of-care testing. Lab Chip 8:1982-1983

Singh AS, Atam V, Yathish BE, Das L, Koonwar S (2014) Role of erythrocyte sedimentation rate in ischemic stroke as an 
inflammatory marker of carotid atherosclerosis. J Neurosci Rural Pract 5:40

Sox HC, Liang MH (1986) Diagnostic decision: the erythrocyte sedimentation rate: guidelines for rational use. Ann Intern Med 104:515-523

Stokes GG (1851) On the effect of the internal friction of fluids on the motion of pendulums, vol 9. Pitt Press, Cambridge

Tilted E, Short E (1999) Clinical utility of the erythrocyte sedimentation rate. Am Fam Physician 60:1443-1450

Tuma RF, Durán WN, Ley K (2011) Microcirculation. Academic Press, London

Uyuklu M, Canpolat M, Meiselman HJ, Baskurt OK (2011) Wavelength selection in measuring red blood cell aggregation based on light transmittance. J Biomed Opt 16:117006-1170069

Viero Y, He Q, Mazenq L, Ranchon H, Fourniols J, Bancaud A (2012) Efficient prototyping of large-scale pdms and silicon nanofluidic devices using pdms-based phase-shift lithography. Microfluid Nanofluidics 12:465-473
Walburn FJ, Schneck DJ (1976) A constitutive equation for whole human blood. Biorheology 13:201-210

Westergren A (1921) Studies of the suspension stability of the blood in pulmonary tuberculosis1. Acta Med Scand 54:247-282

Wintrobe MM, Landsberg JW (1935) A standardized technique for the blood sedimentation test. Am J Med Sci 189:102-114

Xu X, Yu L, Chen Z (2008) Effect of erythrocyte aggregation on hematocrit measurement using spectral-domain optical coherence tomography. IEEE Trans Biomed Eng 55:2753-2758

Yager P, Domingo GJ, Gerdes J (2008) Point-of-care diagnostics for global health. Annu Rev Biomed Eng 10:107-144

Yang Y-A, Lin C-H, Wei Y-C (2014) Thread-based microfluidic system for detection of rapid blood urea nitrogen in whole blood. Microfluid Nanofluidics 16:887-894 